\title{
A Hybrid ARCH-M and BP Neural Network Model For GSCI Futures Price Forecasting
}

\author{
Wen Bo ${ }^{1}$, Wang Shouyang ${ }^{1}$, and K.K. Lai ${ }^{2}$ \\ 1 \\ Institute of System Science, Academy of Mathematics and System Sciences Chinese \\ Academy of Sciences, BeiJing, China \\ wenbo@ams.ac.cn, sywang@ams.ac.cn \\ 2 Department of Management Sciences, City University of Hong Kong \\ Tat Chee Avenue, Kowloon, Hong Kong \\ mskklai@cityu.edu.cn
}

\begin{abstract}
As a versatile investment tool in energy markets for speculators and hedgers, the Goldman Sachs Commodity Index (GSCI) futures are quite well known. Therefore, this paper proposes a hybrid model incorporating ARCH family models and ANN model to forecast GSCI futures price. Empirical results show that the hybrid ARCH(1)-M-ANN model is superior to ARIMA, $\operatorname{ARCH}(1), \operatorname{GARCH}(1,1), \operatorname{EGARCH}(1,1)$ and ARIMA-ANN models on the RMSE, MAPE, Theil IC evaluation criteria.
\end{abstract}

Keywords: ARCH-M, ANN, GSCI, Commodity Index, Forecasting.

\section{Introduction}

Commodity index futures are a versatile tool for taking different forms of exposure to energy commodity markets. They provide investors with an instrument for taking leveraged exposure [1]. There are three famous commodity indices in energy markets which can serve as a surrogate for direct investment: Commodity Research Bureau (CRB) Index, Dow Jones-AIG (DJ-AIG) Commodity Index and Goldman Sachs Commodity Index (GSCI).

CRB index, which began trading on the New York Futures Exchange in 1986, is the oldest. DJ-AIG commodity index, which started in 1999, is rebalanced annually. It reduces return by about $1.5 \%$, compared to monthly rebalancing [2]. GSCI, which started in July 1992 on the Chicago Mercantile Exchange, is a broad-based and production-weighted commodity index. Its volumes have generally exceeded those of CRB index. Lummer and Siegel (1993), who explored GSCI collateralized futures as an asset found that a collateralized position in GSCI futures is a good diversification for stocks and bonds. Paul D.Kaplan and Scott L.Lummer (1997) showed that GSCI collateralized futures were especially valuable to investors who seek diversification and protection against poor returns in other asset classes.

Therefore, we make a research effort covering the GSCI futures, especially the futures price forecasting. In the forecasting field, many researchers have created many successful models, such as ARIMA, ARCH etc... Recently, more hybrid forecasting 
models have been developed that integrate time series model with artificial neural network model to improve prediction accuracy. Lean Yu and Shouyang Wang (2005) obtained a novel nonlinear ensemble forecasting model incorporating GLAR and ANN for foreign exchange rates. G.Peter Zhang (2003) also designed a time series forecasting model using a hybrid ARIMA and a neural networks model. In his paper, he argued that a hybrid methodology can take advantage of the unique strength of ARIMA and ANN models in linear and nonlinear modeling. So this paper proposes a hybrid model incorporating ARCH and ANN model to forecast GSCI futures price.

The rest of the paper is organized as follows. The next section presents a description of the GSCI. The hybrid model is introduced in detail in Section 3. A preliminary analysis and empirical results from the GSCI futures price data sets are reported in Section 4. Conclusions are obtained in Section 5.

\section{Description of the GSCI}

The GSCI is a world production-weighted commodity index comprising of liquid, exchange-traded futures contracts on energy products. It is designed to provide investors with a reliable benchmark for investment performance in energy commodity markets in a manner comparable to the S\&P 500 Index for the stock markets.

The GSCI is world-production weighted; the quantity of each commodity in the index is determined by the average production in the last five years of available data. Such weighting provides the GSCI with significant advantages, both as an economic indicator and as a measure of investment performance. Currently, the GSCI contains 24 commodities from different commodity sectors: six energy products, five industrial metals, eight agricultural products, three livestock products and two precious metals. Table 1 displays a list of the 24 commodities with their dollar weights in the GSCI.

Table 1. GSCI Dollar Weights (\%)

\begin{tabular}{c|c|c|c|c|c}
\hline Commodity & $\begin{array}{c}\text { Dollar } \\
\text { Weights }\end{array}$ & Commodity & $\begin{array}{c}\text { Dollar } \\
\text { Weights }\end{array}$ & Commodity & $\begin{array}{c}\text { Dollar } \\
\text { Weight }\end{array}$ \\
\hline Energy & $\mathbf{7 2 . 9 9}$ & Industrial Metals & $\mathbf{9 . 8 7}$ & Agriculture & $\mathbf{1 0 . 6 2}$ \\
\hline Crude Oil & 30.72 & Aluminum & 3.3 & Wheat (CBOT) & 2.52 \\
\hline Brent Crude oil & 14.57 & Copper & 4.31 & Wheat (KCBT) & 1.05 \\
\hline Unleaded Gas & 8.24 & Lead & 0.28 & Corn & 2.28 \\
\hline Heating Oil & 8.21 & Nickel & 0.88 & Soybeans & 1.36 \\
\hline Gas Oil & 4.5 & Zinc & 1.1 & Cotton & 0.77 \\
\hline Natural Gas & 6.75 & Livestock & $\mathbf{4 . 1 8}$ & Sugar & 1.84 \\
\hline Precious Metals & $\mathbf{2 . 3 4}$ & Live Cattle & 2.08 & Coffee & 0.62 \\
\hline Gold & 2.07 & Feeder Cattle & 0.62 & Cocoa & 0.18 \\
\hline Silver & 0.27 & Lean Hogs & 1.48 & & \\
\hline
\end{tabular}

Source: CME Equity Products: GSCI Components and Weights, 19 May 2006. 
The GSCI futures are listed for all 12 calendar months. The contract value of each GSCI futures position is $\$ 250$ times the GSCI index. The minimum price fluctuation is 0.05 index points, equivalent to a value of $\$ 12.5$ per point. The last trading day of the GSCI futures is the eleventh business day of the contract month, and the position limits is 10,000 net long or short in all contract months combined. The final settlement price shall be the closing price of the GSCI futures price index on the eleventh business day of the contract month.

\section{A Hybrid ARCH-M and BP Neural Network Model}

\subsection{ARCH Family Models}

Following the introduction of autoregressive conditional heteroskedasticity (ARCH) model by Engle (1982), there are many extensions of ARCH model, such as GARCH, GARCH-M, EGACH etc.; we can call them ARCH family models. ARCH model can be expressed as follows

$$
\begin{gathered}
\mathrm{y}_{\mathrm{t}}=\mathrm{x}_{\mathrm{t}}^{\prime} \beta+\varepsilon_{\mathrm{t}} \\
\varepsilon_{t}=\sqrt{h_{t}} \cdot v_{t} \\
h_{t}=\alpha_{0}+\alpha_{1} \varepsilon_{t-1}^{2}+\cdots+\alpha_{q} \varepsilon_{t-q}^{2}
\end{gathered}
$$

Where $v_{t}$ i.i.d., $E\left(v_{t}\right)=0, D\left(v_{t}\right)=1 \quad \alpha_{0}>0, \alpha_{i} \geq 0 \quad \sum_{i=1}^{q} \alpha_{i}<1$

This model is also known as the linear $\operatorname{ARCH}(\mathrm{q})$ model. With financial data it captures the information from $\varepsilon_{\mathrm{t}}$. Bollerslev(1986) proposed an alternative model, the $\operatorname{GARCH}(\mathrm{p}, \mathrm{q})$ model. In his model, $h_{t}$ was expressed as following:

$$
h_{t}=\alpha_{0}+\alpha_{1} \varepsilon_{t-1}^{2}+\cdots+\alpha_{q} \varepsilon_{t-q}^{2}+\theta_{1} h_{t-1}+\cdots+\theta_{p} h_{t-p}
$$

Where $v_{t}$ i.i.d., $E\left(v_{t}\right)=0, D\left(v_{t}\right)=1 \quad \alpha_{0}>0, \alpha_{i} \geq 0 \sum_{i=1}^{q} \alpha_{i}+\sum_{j=1}^{p} \theta_{j}<1$

The ARCH-M model was introduced by Engle, Lilien, and Robins(1987). Since many theories in finance involve an explicit tradeoff between the risk and the expected return, this model is ideally suited to handling such questions in a time series context where the conditional variance may be time-varying. The model is given by

$$
\begin{gathered}
\mathrm{y}_{\mathrm{t}}=\mathrm{x}_{\mathrm{t}}^{\prime} \beta+\gamma \mathrm{h}_{\mathrm{t}}+\varepsilon_{\mathrm{t}} \\
h_{t}=\alpha_{0}+\alpha_{1} \varepsilon_{t-1}^{2}+\cdots+\alpha_{q} \varepsilon_{t-q}^{2}
\end{gathered}
$$

Where $v_{t}$ i.i.d., $E\left(v_{t}\right)=0, D\left(v_{t}\right)=1 \quad \alpha_{0}>0, \alpha_{i} \geq 0 \quad \sum_{i=1}^{q} \alpha_{i}<1$ 
Certainly, the ARCH-M model can also become the GARCH-M model if $\mathrm{h}_{\mathrm{t}}$ fulfill the equation:

$$
h_{t}=\alpha_{0}+\sum_{i=1}^{q} \alpha_{i} \varepsilon_{t-i}^{2}+\sum_{j=1}^{p} \theta_{j} h_{t-j}
$$

People found the applications of the GARCH model limited since the conditional variance is only linked to past conditional variances. Then the EGARCH model was developed by Nelson (1991). In this class of ARCH models, the volatility depended not only on the magnitude of the past surprises in returns but also on their corresponding signs. EGARCH model is given as follows

$$
\begin{gathered}
\ln \left(h_{t}\right)=\alpha_{0}+\sum_{j=1}^{p} \theta_{j} \ln \left(h_{t-j}\right)+\sum_{i=1}^{q} \alpha_{i} g\left(v_{t-i}\right) \\
g\left(v_{t}\right)=\varphi_{i} v_{t}+\left|\frac{\varepsilon_{t}}{\sqrt{h_{t}}}\right|-E\left|\frac{\varepsilon_{t}}{\sqrt{h_{t}}}\right|
\end{gathered}
$$

To sum up, we can find that the ARCH family models focus on building the different models to dispose the variance $\varepsilon_{t}$ in order to obtain more information from the time series data.

\subsection{BP Neural Network Model}

A neural network model takes an input vector $X$ and produces output vector $Y$. The relationship between $X$ and $Y$ is determined by the network architecture. The neural network generally consists of at least three layers: one input layer, one output layer, and one or more hidden layers. It is widely accepted that a three-layer back propagation neural network with an identity transfer function in the output unit and logistic functions in the middle-layer units can approximate well any continuous function arbitrarily, given a sufficient amount of middle-layer units [3].

As Tam and Kiang (1992) reported, the back-propagation algorithm consists of two phases. Suppose we have $s$ samples. Each is described by

$$
\begin{gathered}
x_{i}=\left(x_{i 1}, x_{i 2}, \cdots, x_{i m}\right) \\
T_{i}=\left(t_{i 1}, t_{i 2}, \cdots, t_{i n}\right)
\end{gathered}
$$

Where $X_{i}$ is an input vector, $T_{i}$ is a target output vector and $1 \leq i \leq s$.

In the first phase (forward-propagation), $X_{i}$ is fed into the input layer, and an output $Y_{i}=\left(y_{i 1}, y_{i 2}, \ldots, y_{i n}\right)$ is generated based on the current weight vector $W$. The objective is to minimize an error function $E$, which is defined as

$$
E=\sum_{i=1}^{s} \sum_{j=1}^{n} \frac{\left(y_{i j}-t_{i j}\right)^{2}}{2}
$$


In the second phase (back-propagation), a gradient descent in the weight space, $W$, is performed to locate the optimal solution. The direction and magnitude change $\Delta w_{i j}$ can be computed as

$$
\Delta w_{i j}=-\frac{\partial E}{\partial w_{i j}} \varepsilon
$$

Where $0<\mathcal{E}<1$ is a parameter controlling the algorithm's convergence rate.

\subsection{A Hybrid Model}

Since it is difficult to completely know the characteristics of the data in a real problem, hybrid methodology that has both linear and nonlinear capabilities can be a good strategy for practical use. According to the theory of $\mathrm{ARCH}$ model, we have the LM to test the GSCI data, and we find that the GSCI data have the ARCH effects. Therefore, we propose a hybrid model incorporating ARCH model and ANN model.

Considering a time series to be composed of a linear relation structure and a nonlinear component [4], the hybrid model is

$$
\hat{\mathrm{Y}}_{t}=\hat{L}_{t}+\hat{N}_{t}
$$

Where $\hat{L}_{t}$ and $\hat{N}_{t}$ denote the linear and nonlinear components.

We first process the ARCH family model for the linear component, and then we obtain the residuals. The residual at time $t$ is

$$
e_{t}=y_{t}-\hat{L}_{t}
$$

With the ANN model, the residual forecast value $\hat{N}_{t}$ will be

$$
\hat{N}_{t}=f\left(e_{t-1} e_{t-2}, \cdots \ldots e_{t-n}\right)+\xi_{t}
$$

Where $f(\cdot)$ is a nonlinear function and $\xi_{t}$ is the random error.

Thus, the combined forecast will be

$$
\hat{\mathrm{Y}}_{t}=\hat{L}_{t}+\hat{N}_{t}
$$

\subsection{Forecast Evaluation Criteria}

Three criteria will be used to evaluate the model forecasting ability. The first is the root mean squared error (RMSE). The formula for RMSE is

$$
\operatorname{RMSE}=\sqrt{\frac{\sum\left(\hat{\mathrm{y}}_{\mathrm{t}}-\mathrm{y}_{\mathrm{t}}\right)^{2}}{\mathrm{n}}}
$$

Where $\hat{y}_{t}$ is the predicted value, $\mathrm{y}_{\mathrm{t}}$ is the actual value. 
The second is the mean absolute percent error (MAPE). It is a measure of average error in percentage terms for each point forecast. MAPE is given by

$$
\mathrm{MAPE}=\frac{1}{\mathrm{n}} \sum\left|\frac{\hat{\mathrm{y}}_{\mathrm{t}}-\mathrm{y}_{\mathrm{t}}}{\mathrm{y}_{\mathrm{t}}} \times 100\right|
$$

The third is the Theil Inequality Coefficient (Theil IC). Theil IC value is always between 0 and 1 , and a smaller value indicates the error between the predicted value and the actual value is smaller. Theil IC is given by

$$
\text { Theil IC }=\frac{\sqrt{\frac{\sum\left(\hat{y}_{t}-y_{t}\right)^{2}}{n}}}{\sqrt{\frac{\sum \hat{y}_{t}^{2}}{n}}+\sqrt{\frac{\sum y_{t}^{2}}{n}}}
$$

\section{Empirical Analysis}

\subsection{Data Description}

The daily GSCI futures price data are obtained from Reuters Information System. The data span the period from 8 July 1996 to 26 May 2006 (2488 observations). We take data from 8 July 1996 to 25 May 2005 as in-sample data sets ( 2236 observations), and we take the data from 27 May 2005 to 26 May 2006 as out-of-sample data sets ( 252 observations) which are used to evaluate the performance of the predictions. Figure1 plots logarithm returns data series of GSCI futures price.

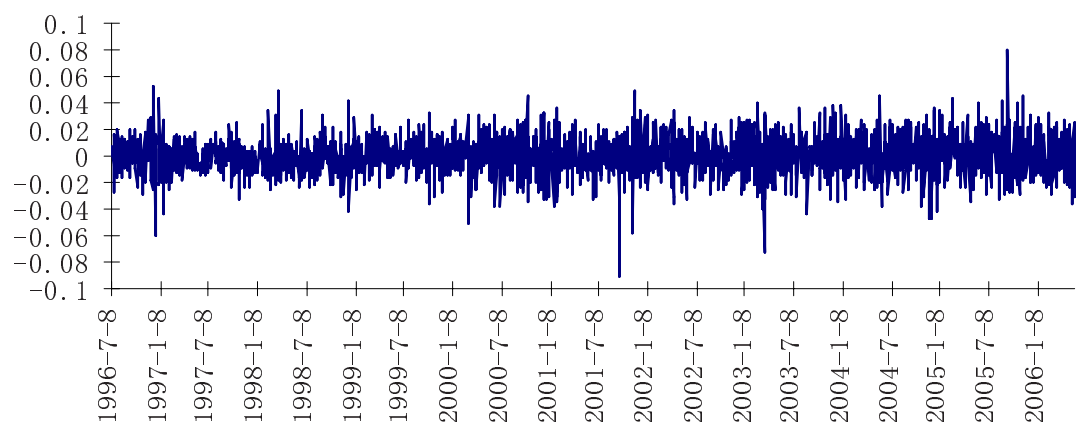

Fig. 1. Logarithm returns data series of GSCI futures price

\subsection{Empirical Results}

In this study, Arima and ARCH family models are implemented via the Eviews software, which is produced by Quantitative Micro Software Corporation. ANN model is built using the Matlab software, which is produced by Mathworks 
Laboratory Corporation. The main equation is regression equation and Akaike's Information Criterion (AIC) rule is used to determine the lag lengths. Finally, we select 3 lags after testing many times. The residual equation we selected are ARCH (1), GARCH (1,1), ARCH (1)-M, GARCH (1,1)-M, EGARCH(1,1) for Bollerslev's (1988) idea, in most applications $\mathrm{p}=\mathrm{q}=1$ is found to suffice. On the other hand, the ANN model uses trial and error to determine the network architecture of 4-4-1 by minimizing the forecasting error [5].

First, we process the forecast using the ARCH family models. Table 2 gives the forecasting result. According to the forecast evaluating criteria, we can find that the ARCH(1)-M performs best.

Table 2. Forecast Result of ARCH Family Models

\begin{tabular}{c|c|c|c|c|c|c}
\hline ARCH Family & RMSE & Rank & MAPE & Rank & Theil IC & Rank \\
\hline Arima & 7.3347 & 6 & 1.3650 & 5 & 0.008486 & 6 \\
\hline ARCH(1) & 7.3303 & 3 & 1.3651 & 6 & 0.00848 & 3 \\
\hline GARCH(1,1) & 7.3317 & 4 & 1.3649 & 4 & 0.008482 & 4 \\
\hline EGARCH(1,1) & 7.3331 & 5 & 1.3648 & 3 & 0.008485 & 5 \\
\hline ARCH(1)-M & 7.3241 & 1 & 1.3571 & 1 & 0.008476 & 1 \\
\hline GARCH(1,1)-M & 7.3257 & 2 & 1.3620 & 2 & 0.008478 & 2 \\
\hline
\end{tabular}

Therefore, we form the hybrid model using the best ARCH(1)-M model combined with the ANN model. Table 3 gives the forecasting results. Simultaneously, we also process the forecast using the ARIMA-ANN hybrid model. According to the forecast evaluation criteria, we can find that the ARCH(1)-M-ANN model performs better than the ARIMA-ANN model, and also performs the best in all the given models.

Table 3. Forecast Result of Hybrid Models

\begin{tabular}{c|c|c|c|c|c|r}
\hline Hybrid Models & RMSE & Rank & MAPE & Rank & Theil IC & Rank \\
\hline ARCH(1)-M-ANN & 7.2347 & 1 & 1.3376 & 1 & 0.008371 & 1 \\
\hline Arima-ANN & 7.3334 & 2 & 1.3593 & 2 & 0.008485 & 2 \\
\hline
\end{tabular}

According to the NMSE, MAPE, Thiel IC evaluation criteria, we can find that the $\operatorname{ARCH}(1)-M-A N N$ hybrid model is superior to $\operatorname{ARIMA}, \operatorname{ARCH}(1), \operatorname{GARCH}(1,1)$, EGARCH(1,1), ARCH(1)-M , GARCH(1,1)-M and ARIMA-ANN models for GSCI futures price forecasting.

\section{Conclusions}

Commodity index futures represent a useful investment vehicle for speculators and hedgers in energy markets. In this study, we propose a hybrid model combing ARCH$\mathrm{M}$ model and ANN model to predict GSCI futures price. In terms of the empirical 
results, we find that the model combining ARCH-M and ANN models performs the best on the selected criteria.

Acknowledgements. This work is supported by City University of Hong Kong. Project Number: 9610058.

\section{References}

1. Wai Mun Fong, Kim Hock See. Modelling the conditional volatility of commodity index futures as a regime switching process. Journal of Applied Econometrics 2001; 16: pp. 133163.

2. Commodity indexes overview and analysis. Seamans Capital Management. July 2003.

3. H. White. Connectionist nonparametric regression: multilayer feedforward networks can learn arbitrary mappings. Neural Networks, 1990, 3: pp. 535-549.

4. G.Peter Zhang. A time series forecasting using a hybrid ARIMA and neural network model. Neurocomputing. 2003; 50: pp.159-75.

5. Lean Yu, Shouyang Wang. A novle nonlinear ensemble forecasting model incorporating GLAR and ANN for foreign exchange rates. Computers \& Operations Research 2005; 32.

6. Tim Bollerslev, Ray Y. Chou, Kenneth F. Kroner. ARCH modeling in finance. Journal of Econometrics 1992; 52: pp. 5-59.

7. Nowrouz Kohzadi, Milton S. Boydd. A comparison of artificial neural network and time series models for forecasting commodity prices. Neurocomputing 1996; 10:pp. 169-181.

8. G.E.P. Box. Time series analysis. Forecasting and Control.San Francisco, CA, 1970.

9. CME GSCI Futures and Options 2006 Information Guide. CME Equity Products.2006.

10. Sayantani Ghose. Downside risk in the DJ-AIG commodity index futures market. Working paper Submitted to European Journal of Finance. 2005.

11. Lummer, Siege.GSCI Collterized Futures: A hedging and diversification tool for institutional investors. Journal of Investing. 1993.

12. Engle, Robert F. Autoregressive conditional heteroskedasticity with estimates of variance of the U.K. inflation. Econometrica. 1982, 50: pp. 987-1008.

13. Paul D.Kaplan. GSCI collateralized futures as a hedging and diversification tool for institutional portfolios: an update. Forthcoming in the Journal of Investing 1997; 12. 